\title{
Will Palliative Care Ever Be Cool?
}

\author{
Brian Badgwell, MD, MS \\ MD Anderson Cancer Center, Houston, TX
}

In an article that accompanies this editorial, the authors acknowledge that palliative care may still carry a somewhat negative stigma, but report an amazing $74 \%$ rate of use of physician palliative care services. Therefore, in answer to the question in the title, YES! Palliative care can be cool—in Canada. Let us not forget that Canada provided many early contributions to the hospice and palliative care movement, and no mention of surgery and palliative care is complete without acknowledging Dr. Balfour Mount, a surgeon often considered the father of palliative care in North America. Recognizing Canada's importance in the dissemination of palliative care has helped me personally deal with some of their musical exports, such as Justin Bieber and Nickelback. For a more recent perspective, Palliative Care Matters, a national initiative to achieve a consensus on ensuring high-quality palliative care as part of Canada's universal healthcare model, can provide some information on utilization and public opinion of palliative care. Awareness of palliative care was found in $58 \%$ of Canadians, with $51 \%$ having had someone important to them receive palliative care within the past 10 years, of whom $89 \%$ were satisfied with the care. ${ }^{1}$

A considerable number of studies have been published demonstrating that palliative care can result in, or is at least associated with, less aggressive end-of-life care. ${ }^{2-6}$ More importantly, the integration of palliative care in advanced cancer patients can result in improved quality of life and decreased symptom burden. ${ }^{7}$ Palliative care may even be associated with improved survival, and it is not shocking that improved quality of life can also lead to improved length of life., ${ }^{2,8}$ Increasing recognition of these

(C) Society of Surgical Oncology 2018

First Received: 30 April 2018;

Published Online: 10 May 2018

B. Badgwell, MD, MS

e-mail: bbadgwell@mdanderson.org associations has led the American Society of Clinical Oncology (ASCO) to recommend dedicated palliative care services, early in the disease course, concurrent with active treatment. ${ }^{9,10}$

One limitation that has plagued studies searching for an association between palliative care and less-aggressive care is the inherent bias of the palliative care consultation. Patients referred for palliative care services may have been deemed less optimal for aggressive care, with the palliative care consultation a result of the trajectory of the patient's clinical course. Alternately, and more likely, the palliative care services generated the trajectory of less-aggressive care. One method to eliminate this potential bias is to limit the cohort to patient's only receiving a palliative care consultation, and evaluate the timing of palliative care intervention. Preliminary evidence indicates that early palliative care consultation, compared with later consultation, can also result in less-aggressive end-of-life care, and therefore provides further evidence supporting the impact of palliative care. ${ }^{3}$

Palliative care has done a fantastic job at justifying itself as a specialty, and also as an important tool in improving patients quality of life and symptom control. But what is in the palliative care syringe, and what is in this syringe from Toronto? Identification of the key elements of palliative care that are associated with improved outcomes would allow for education of oncologists, streamlining of palliative care, and dissemination across the cancer care trajectory. Investigators in some of the landmark studies demonstrating the effectiveness of palliative care have now gone on to try to identify these key elements. Treatment that focuses on coping, decision making, and advanced care planning was found in association with improved patient-reported outcomes. ${ }^{11}$ The key elements found in the study by Merchant et al. ${ }^{12}$ are not obtainable from such large, population-based databases, but are limited to fee 
code descriptions such as case management fees, counseling of relatives of a terminally ill patient, and palliative care support.

Dissemination of palliative care is needed in oncology as many patients want to receive care at home at the end of their life, but also to meet the challenge of providing palliative care in combination with oncology care. Palliative care in the hospital has an important role in symptom management and counseling on goals of care, but outpatient care is needed for early integration and also to provide effective continuous palliative care integrated with standard oncology care. Patients with advanced cancer will experience symptom changes and palliative care needs that will need to be addressed by their primary physicians and oncologists. We are scratching the surface in identifying what are the most important components of palliative care, but are in desperate need of a deeper dive to assist in training the current and future workforce to provide basic palliative care and utilize specialists appropriately.

Surgical oncology has an even more difficult path forward in that there are limited studies that have identified the palliative care needs of surgical patients. There is no specific consensus statement from the Society of Surgical Oncology regarding integration of palliative care, likely because we fit under the ASCO statement. The American College of Surgeons Committee on Surgical Palliative Care has embraced the challenge of integrating palliative care principles into the management of patients and families requiring surgical care in the setting of advanced disease. This committee's mission is to incorporate the techniques of palliative care into practice, with a focus on education of the general surgical workforce. With contributions such as that from Merchant et al. ${ }^{12}$ and investigators and societies interested in identifying actionable components of palliative care that can be disseminated, hopefully we will not stop at recognizing what is in the syringe of palliative care, but also know when to use the knife of palliative surgical care.

\section{REFERENCES}

1. Roulston E. Canadians' Views on Palliative Care. J Palliat Med. 2018; 21(S1):S9-14.

2. Temel JS, Greer JA, Muzikansky A, et al. Early palliative care for patients with metastatic non-small-cell lung cancer. $N$ Engl $J$ Med. 2010; 363(8):733-42.

3. Hui D, Kim SH, Roquemore J, et al. Impact of timing and setting of palliative care referral on quality of end-of-life care in cancer patients. Cancer. 2014; 120(11):1743-9.

4. Wiesenthal A, Goldman DA, Korenstein D. Impact of Palliative Medicine Involvement on End-of-Life Services for Patients With Cancer With In-Hospital Deaths. J Oncol Pract. 2017; 13(9):e749-59.

5. Triplett DP, LeBrett WG, Bryant AK, et al. Effect of Palliative Care on Aggressiveness of End-of-Life Care Among Patients With Advanced Cancer. J Oncol Pract. 2017; 13(9):e760-9.

6. Henson LA, Gomes B, Koffman J, et al. Factors associated with aggressive end of life cancer care. Support Care Cancer. 2016; 24(3):1079-89.

7. Vanbutsele G, Pardon K, Van Belle S, et al. Effect of early and systematic integration of palliative care in patients with advanced cancer: a randomised controlled trial. Lancet Oncol. 2018; 19(3):394-404

8. Bakitas MA, Tosteson TD, Li Z, et al. Early Versus Delayed Initiation of Concurrent Palliative Oncology Care: Patient Outcomes in the ENABLE III Randomized Controlled Trial. J Clin Oncol. 2015; 33(13):1438-45.

9. Ferrell BR, Temel JS, Temin S, et al. Integration of Palliative Care Into Standard Oncology Care: ASCO Clinical Practice Guideline Update Summary. J Oncol Pract. 2017; 13(2):119-21.

10. Ferrell BR, Temel JS, Temin S, et al. Integration of Palliative Care Into Standard Oncology Care: American Society of Clinical Oncology Clinical Practice Guideline Update. J Clin Oncol. 2017; 35(1):96-112.

11. Hoerger M, Greer JA, Jackson VA, et al. Defining the Elements of Early Palliative Care That Are Associated With Patient-Reported Outcomes and the Delivery of End-of-Life Care. J Clin Oncol. 2018; 36(11):1096-102.

12. Merchant SJ, Brogly SB, Goldie C, et al. Palliative Care is Associated with Reduced Aggressive End-of-Life Care in Patients with Gastrointestinal Cancer. Ann Surg Oncol. 2018; 25(6):1478-87. 